\title{
Evaluation of the 2015 AGA guidelines on pancreatic cystic neoplasms in a large surgically confirmed multicenter cohort
}

\section{다)(웅}

\section{Authors}

Phillip S. Ge1 , V. Raman Muthusamy¹, Srinivas Gaddam³ , DianaMarie Jaiyeola ${ }^{4}$, Stephen Kim ${ }^{1}$, Alireza Sedarat ${ }^{1}$, Timothy R. Donahue ${ }^{2}$, Lindsay Hosford ${ }^{5}$, Robert H. Wilson ${ }^{5}$, David P. Grande ${ }^{4}$, Rajesh N. Keswani ${ }^{4}$, Vladimir M. Kushnir ${ }^{3}$, Daniel Mullady ${ }^{3}$, Steven A. Edmundowicz ${ }^{5}$, Dayna S. Early ${ }^{3}$, Srinadh Komanduri ${ }^{4}$, Sachin Wani ${ }^{5}$, Rabindra R. Watson ${ }^{1}$

\section{Institutions}

1 Division of Digestive Diseases and Department of Surgery, David Geffen School of Medicine at UCLA, Los Angeles, California, United States

2 University of California Los Angeles David Geffen School of Medicine - Department of Surgery, Los Angeles, California, United States

3 Division of Gastroenterology, Washington University in St. Louis School of Medicine, St. Louis, Missouri, United States

4 Division of Gastroenterology, Northwestern Memorial Hospital, Chicago, Illinois, United States

5 Division of Gastroenterology and Hepatology, University of Colorado Anschutz Medical Campus, Aurora, Colorado, United States

submitted 13.9.2016

accepted after revision $\quad$ 17.11.2016

\author{
Bibliography \\ DOI http://dx.doi.org/10.1055/s-0042-122010 | \\ Endoscopy International Open 2017; 05: E201-E208 \\ (c) Georg Thieme Verlag KG Stuttgart · New York \\ ISSN 2364-3722
}

Corresponding author

Rabindra R. Watson, MD, Division of Digestive Diseases, David Geffen School of Medicine at UCLA, 200 UCLA Medical Plaza,

Suite 365A, Los Angeles, CA 90095

Fax: +1-310-206-0007

rwatson@mednet.ucla.edu

\section{ABSRACT}

Background and study aims The American Gastroenterological Association (AGA) recently published guidelines for the management of asymptomatic pancreatic cystic neoplasms (PCNs). We aimed to evaluate the diagnostic characteristics of the AGA guidelines in appropriately recommending surgery for malignant PCNs. Patients and methods A retrospective multicenter study was performed of patients who underwent endoscopic ultrasound (EUS) for evaluation of PCNs who ultimately underwent surgical resection from 2004-2014. Demographics, EUS characteristics, fine-needle aspiration (FNA) results, type of resection, and final pathologic diagnosis were recorded. Patients were categorized into 2 groups (surgery or surveillance) based on what the AGA guidelines would have recommended. Performance characteristics for the diagnosis of cancer or high-grade dysplasia (HGD) on surgical pathology were calculated.

Results Three hundred patients underwent surgical resection for PCNs, of whom the AGA guidelines would have recommended surgery in 121 (40.3\%) and surveillance in 179 (59.7\%) patients. Among patients recommended for surgery, 45 (37.2\%) had cancer, whereas 76 (62.8\%) had no cancer/HGD. Among patients recommended for surveillance, 170 (95.0\%) had no cancer/HGD; however, $9(5.0 \%)$ patients had cancer that would have been missed. For the finding of cancer/HGD on surgical pathology, the AGA guidelines had $83.3 \%$ sensitivity ( $95 \%$ Cl $70.7-92.1$ ), $69.1 \%$ specificity $(95 \% \mathrm{Cl} 62.9-74.8), 37.2 \%$ positive predictive value $(95 \% \mathrm{Cl}$ 28.6 - 46.4), $95.0 \%$ negative predictive value ( $95 \%$ Cl 90.7-97.7), and $71.7 \%$ accuracy ( $95 \% \mathrm{Cl} 67.4-74.6)$.

Conclusions The 2015 AGA guidelines would have resulted in $60 \%$ fewer patients being referred for surgical resection, and accurately recommended surveillance in $95 \%$ of patients with asymptomatic PCNs. Future prospective studies are required to validate these guidelines.

Meeting presentations: Presented in part at Digestive Diseases Week 2016

\section{Introduction}

Pancreatic cystic neoplasms (PCNs) represent a heterogeneous group of lesions with varying malignant potential. There is significant variation in the reported prevalence of PCNs, ranging from $<1 \%$ to $24.3 \%$, however, they are increasingly being incidentally detected due to widespread use of cross-sectional imaging including computed tomography $(\mathrm{CT})$ and magnetic resonance imaging (MRI) [1,2].
PCNs present a significant management dilemma. Certain lesions such as serous cystadenomas (SCAs) exhibit very low malignant potential. On the other hand, mucinous cystic neoplasms (MCNs), branch-duct intraductal papillary mucinous neoplasms (IPMNs), and main-duct IPMNs are reported to harbor malignant potential of $10 \%$ to $20 \%, 15 \%$ to $25 \%$, and $40 \%$ to $50 \%$, respectively [3-5]. Although surgical resection is curative in the absence of invasive carcinoma, surgery can also be associated with significant morbidity and potential mortality [6]. 
- Table 1 Comparison of Sendai (2006), Fukuoka (2012), and AGA (2015) guidelines on management of pancreatic cystic neoplasms.

\begin{tabular}{|c|c|c|c|}
\hline Issues & 2006 Sendai guidelines & 2012 Fukuoka guidelines & 2015 AGA guidelines \\
\hline \multirow[t]{5}{*}{ Surveillance } & Cyst< $1 \mathrm{~cm}$ : MRI annually & Cyst $>3 \mathrm{~cm}$ without high-risk stigmata ${ }^{1}$ or & Less than 2 high-risk features ${ }^{2}$ : repeat MRI \\
\hline & $\begin{array}{l}\text { Cyst } 1-2 \mathrm{~cm} \text { without high- } \\
\text { risk stigmata } \text { : surveillance } \\
\text { every } 6-12 \text { months }\end{array}$ & $\begin{array}{l}\text { worrisome features }{ }^{4} \text { : alternate EUS with } \\
\text { MRI every } 3-6 \text { months }\end{array}$ & $\begin{array}{l}\text { in } 1 \text { year, then every } 2 \text { years until year } 5 \text {, then } \\
\text { stop surveillance }\end{array}$ \\
\hline & $\begin{array}{l}\text { Cyst } 2-3 \mathrm{~cm} \text { without high- } \\
\text { risk stigmata }{ }^{3} \text { : surveillance } \\
\text { every } 3-6 \text { months }\end{array}$ & $\begin{array}{l}\text { Cyst } 2-3 \mathrm{~cm} \text { without high-risk stigmata }{ }^{1} \text { or } \\
\text { worrisome features }{ }^{4} \text { EUS in } 3-6 \text { months } \\
\text { then lengthen interval and alternate with } \\
\text { MRI }\end{array}$ & $\begin{array}{l}\text { Two or more high-risk features }{ }^{2} \text { : EUS-FNA, if } \\
\text { negative, then repeat MRI in } 1 \text { year, then every } \\
2 \text { years until year } 5 \text {, then stop surveillance }\end{array}$ \\
\hline & & $\begin{array}{l}\text { Cyst } 1-2 \mathrm{~cm} \text { without high-risk stigmata }{ }^{1} \text { or } \\
\text { worrisome features }{ }^{4} \mathrm{CT} / \mathrm{MRI} \text { yearly for } 2 \\
\text { years, then lengthen interval if no change }\end{array}$ & $\begin{array}{l}\text { Discontinue surveillance when patient is no } \\
\text { longer a surgical candidate }\end{array}$ \\
\hline & & Cyst $<1 \mathrm{~cm}: \mathrm{CT} / \mathrm{MRI}$ in $2-3$ years & \\
\hline \multirow[t]{4}{*}{ Surgical referral } & Cyst $>3 \mathrm{~cm}$ & Any high-risk stigmata ${ }^{1}$ & $\begin{array}{l}\text { At least } 2 \text { high-risk features }{ }^{2} \text { confirmed by } \\
\text { EUS-FNA, or concerning cytology }\end{array}$ \\
\hline & $\begin{array}{l}\text { Cyst } 1-3 \mathrm{~cm} \text { with any } \\
\text { high-risk stigmata }^{3}\end{array}$ & Worrisome features ${ }^{3}$ on EUS & \\
\hline & & Cyst $>3 \mathrm{~cm}$ in young/fit patients & \\
\hline & & Cyst $2-3 \mathrm{~cm}$ in young/fit patients & \\
\hline Main duct IPMN & Surgical resection & Surgical resection & Not evaluated \\
\hline \multirow{3}{*}{$\begin{array}{l}\text { Postoperative } \\
\text { surveillance }\end{array}$} & No cancer: no surveillance & MCN: no surveillance & No cancer or HGD: no surveillance \\
\hline & $\begin{array}{l}\text { Cancer: MRI or CT every } \\
6 \text { months }\end{array}$ & $\begin{array}{l}\text { IPMN with positive margins: MRI every } \\
6 \text { months }\end{array}$ & Cancer or HGD: MRI every 2 years \\
\hline & & $\begin{array}{l}\text { IPMN with negative margins: MRI at } 2 \text { and } \\
5 \text { years }\end{array}$ & \\
\hline \multicolumn{4}{|c|}{$\begin{array}{l}\text { AGA, American Gastroenterological Association; CT, computed tomography; EUS-FNA, endoscopic ultrasonography with fine-needle aspiration; HGD, high-grade } \\
\text { dysplasia; IPMN, intraductal papillary mucinous neoplasm; MRl, magnetic resonance imaging } \\
{ }^{1} \text { High-risk stigmata (Fukuoka 2012): obstructive jaundice, solid component, dilated main pancreatic duct } \geq 10 \mathrm{~mm} \\
{ }^{2} \text { High-risk features (AGA 2015): cyst } \geq 3 \mathrm{~cm} \text {, solid component, dilated main pancreatic duct } \\
{ }^{3} \text { High-risk stigmata (Sendai 2006): mural nodules, dilated main pancreatic duct, positive cytology } \\
{ }^{4} \text { Worrisome features (Fukuoka 2012): pancreatitis, cyst } \geq 3 \mathrm{~cm} \text {, thickened cyst wall, main pancreatic duct } 5-9 \mathrm{~mm} \text {, nonenhancing mural nodule, abrupt change in } \\
\text { caliber of pancreatic duct with distal pancreatic atrophy }\end{array}$} \\
\hline
\end{tabular}

Endoscopic ultrasound (EUS) is increasingly utilized due to its ability to provide valuable information in differentiating mucinous from non-mucinous PCNs and identify malignant cysts [7]. Certain EUS features have been shown to correlate with malignant potential, such as presence of a solid component or mural nodule, thickened cyst walls, and a dilated main pancreatic duct [8]. EUS also allows for fine-needle aspiration (FNA) for cyst fluid analysis and cytology, the latter of which can be highly specific for detection of malignancy [9]. Various studies have also evaluated the utility of cyst fluid tumor markers such as carcinoembryonic antigen (CEA) in diagnosis of mucinous or pre-malignant cysts [9].

However, despite these apparent advantages, EUS morphology, cyst fluid analysis, and cytologic analysis are each insufficient alone to diagnose potentially malignant lesions [9-12]. Multiple novel biomarkers have been evaluated such as microRNA as well as somatic mutations in the GNAS and KRAS genes and appear promising in accurate detection of potentially malignant lesions; however, these tests have yet to be fully va- lidated for routine clinical use $[13,14]$. Due to the uncertainties regarding the management of PCNs, multiple consensus guidelines have been developed, including the Sendai guidelines in 2006, Fukuoka guidelines in 2012, and European guidelines in 2013 [15-17]. While these guidelines have been validated in various studies [18-20], application of these guidelines has been shown to result in unnecessary surgery for many benign low-risk cysts, while missing other cysts with high-grade dysplasia or associated invasive cancer [19, 21-24].

Recently, the American Gastroenterological Association (AGA) published guidelines for the management of asymptomatic PCNs $[25,26]$. The 2015 AGA guidelines call for a much less aggressive surveillance regimen than the Sendai and Fukuoka guidelines ( $\triangleright$ Table $\mathbf{1}$ ). In this study, we seek to evaluate the diagnostic performance of the AGA guidelines for identification of high-grade dysplasia (HGD) or cancer in patients with PCNs who had previously undergone EUS with or without FNA followed by surgical resection. 


\section{Patients and methods}

\section{Participating centers}

We performed a retrospective cohort study at four academic tertiary care referral centers with extensive pancreatic surgical experience and interest in pancreatic cystic neoplasms. These include the Ronald Reagan University of California Los Angeles Medical Center (Los Angeles, CA), the Barnes-Jewish Hospital at Washington University in St. Louis (St. Louis, MO), the University of Colorado Anschutz Medical Center (Aurora, CO), and Northwestern Memorial Hospital (Chicago, IL). Institutional Review Board approval was obtained at each institution.

\section{Inclusion and exclusion criteria}

We included all patients who were evaluated for a PCN with EUS with or without FNA from 2004-2014 who subsequently underwent surgical resection. Prior to 2006, participating institutions referred patients to surgery based on interpretation of cross-sectional imaging, EUS morphologic characteristics, and cyst fluid analysis. Each of the participating centers subsequently incorporated the Sendai guidelines from 2006-2012, and the Fukuoka guidelines from 2012 until the time of this study analysis.

We excluded patients with PCNs who were referred directly to surgery without undergoing EUS; patients who underwent surgery for non-neoplastic pancreatic cysts such as pseudocysts; patients who underwent surgery for cystic lesions that were specifically not evaluated in the AGA guidelines such as main duct IPMNs, solid pseudopapillary neoplasms, cystic neuroendocrine tumors, and cystic degeneration of pancreatic ductal adenocarcinomas; and patients who underwent serial surveillance with EUS or cross-sectional imaging who did not ultimately undergo surgery. Finally, patients were excluded if data could not be accurately obtained from review of their records.

\section{Demographics and clinical history}

For all patients meeting inclusion criteria, electronic medical records were reviewed. EUS was performed according to the current standard of care by experienced endosonographers. FNA samples were obtained using a variety of endoscopic needles at the discretion of the endosonographer. Surgical data were collected by review of operative reports. FNA and surgical pathology data were obtained by review of the final pathology reports generated by a gastrointestinal pathologist.

Data collected included basic demographics including age and sex, presence of high-risk features on EUS as defined by the AGA guidelines (cyst size $\geq 3 \mathrm{~cm}$, presence of solid cyst component, dilated main pancreatic duct), FNA cytology results including mucin or cellular atypia, type of surgical resection, and final diagnosis on surgical pathology. While the AGA guidelines do not explicitly define "dilated main pancreatic duct," for the purposes of this study we utilized a cut-off of $\geq 5 \mathrm{~mm}$, as used to define "worrisome feature" in the Fukuoka guidelines [16]. Cytology specimens were considered "concerning" if there was

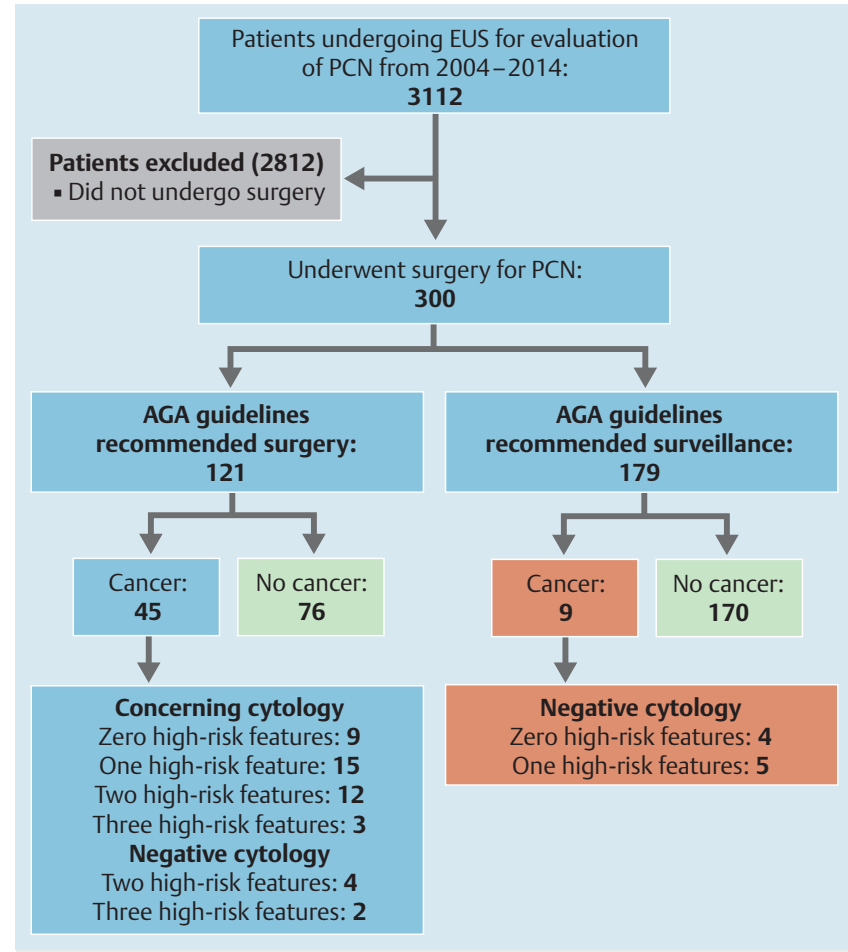

Fig. 1 Flowchart of patient selection and major study results.

evidence of cytologic atypia, high-grade dysplasia, or adenocarcinoma.

\section{Study outcomes and statistical analysis}

Patients were categorized into 2 groups (surgery or surveillance) based on what the AGA guidelines would have recommended. Our aim was to evaluate performance characteristics of the AGA guidelines for identification of PCN with HGD or cancer.

At each of the participating centers, data from electronic medical records were entered into a password-protected, deidentified Microsoft Access database (Microsoft Corporation, Redmond, WA). The databases were transferred to a centralized location where the data were merged, compared, and reconciled for accuracy. Descriptive statistics were used to characterize the demographics of our population. Continuous data were summarized using means and standard deviation, and nominal data were summarized using counts and percentages. We subsequently calculated performance characteristics of the AGA guidelines for the finding of cancer or high-grade dysplasia on surgical pathology, including sensitivity, specificity, diagnostic accuracy, positive and negative predictive values, and positive and negative likelihood ratios.

\section{Results}

A flow chart depicting our results and major findings is shown in $>$ Fig. 1. 


\begin{tabular}{|c|c|c|}
\hline & & Incidence (n, \%) \\
\hline \multirow[t]{2}{*}{ Demographics } & Total patients & 300 \\
\hline & Age (years, mean $\pm S D$ ) & $62.6 \pm 13.8$ \\
\hline \multirow[t]{2}{*}{ Gender } & Male & $113(37.7)$ \\
\hline & Female & $187(62.3)$ \\
\hline \multirow[t]{5}{*}{ Surgery } & Whipple & $127(42.5)$ \\
\hline & Distal pancreatectomy & $154(51.5)$ \\
\hline & Middle pancreatectomy & $9(3.0)$ \\
\hline & Total pancreatectomy & $8(2.7)$ \\
\hline & Exploratory Llparotomy & $1(0.3)$ \\
\hline \multirow[t]{7}{*}{ Cyst findings } & IPMN without cancer & $147(49.0)$ \\
\hline & IPMN with cancer & $49(16.3)$ \\
\hline & $\begin{array}{l}\text { IPMN with high-grade } \\
\text { dysplasia (carcinoma-in-situ) }\end{array}$ & $2(0.7)$ \\
\hline & MCN without cancer & $60(20.0)$ \\
\hline & MCN with cancer & $3(1.0)$ \\
\hline & SCA without cancer & $39(13.0)$ \\
\hline & SCA with cancer & $0(0.0)$ \\
\hline \multirow[t]{4}{*}{ Cancer } & Total cancer & $54(18.0)$ \\
\hline & IPMN with adenocarcinoma & $49(90.7)$ \\
\hline & $\begin{array}{l}\text { IPMN with high grade } \\
\text { dysplasia }\end{array}$ & $2(3.7)$ \\
\hline & MCN with adenocarcinoma & $3(5.6)$ \\
\hline
\end{tabular}

\section{Patient characteristics}

A total of 3112 patients underwent EUS for evaluation of a PCN during the study period, of whom 300 patients were identified as having undergone surgical resection for PCNs relevant to the AGA guidelines. Patient demographics are shown in $\mathbf{T a b l e} 2$. Among the 300 patients who underwent surgical resection, the average age was 65.2 years and $62.3 \%$ were female.

\section{EUS/surgical details}

Characteristics of surgical resection are shown in Table 3. A total of 11/300 (3.7\%) patients had all 3 high-risk features defined in the AGA guidelines, $42 / 300$ (14.0\%) patients had 2 high-risk features, $113 / 300(37.7 \%)$ patients had 1 high-risk feature, and 134/300 (44.6\%) patients had no high-risk features. A total of 98/300 (34.7\%) patients had concerning cytology findings such as atypia or malignancy, of whom 9 (9.2\%) patients had 3 high-risk features, 21 (21.4\%) patients had 2 high-risk features, 36 (36.7\%) patients had 1 high-risk feature, and $32(32.7 \%)$ patients had no high-risk features.

Surgical resection included Whipple pancreaticoduodenectomy (127/300, 42.5\%), distal pancreatectomy (154/300,
$51.5 \%)$, middle pancreatectomy (9/300, 3.0\%), total pancreatectomy $(8 / 300,2.7 \%)$, and exploratory laparotomy with surgical biopsy $(1 / 300,0.3 \%)$. The surgical pathology for resected cystic neoplasms included MCNs (63/300, 21.0\%), IPMNs (198/300, 66.0\%), and serous cystadenomas (39/300, 13.0\%). Among surgically resected lesions, none of the 39 serous cystadenomas contained adenocarcinoma, while 49/198 (24.7\%) IPMNs contained adenocarcinoma, 2/198 (1.0\%) IPMNs contained HGD, and 3/63 (4.8\%) MCNs contained adenocarcinoma. Serous cystadenomas underwent surgical resection for a variety of reasons which included unclear diagnosis on preoperative EUS, and suspicion of a solid mass in 3 patients.

A total of 54 patients were found to have malignant PCNs, and their characteristics are shown in Table 4. Among these patients, 45/54 (83.3\%) either had evidence of concerning cytology or had 2 or more high-risk features and would have been recommended for surgery, whereas 9/54 (16.7\%) had no evidence of concerning cytology and fewer than 2 high-risk features and would have been recommended for surveillance. Of note, 15 patients had evidence of concerning cytology with only 1 high-risk feature, and 9 patients had evidence of concerning cytology only without any high-risk features; had the AGA guidelines been followed, these 24 patients would have been recommended for surgery based solely on concerning cytology.

Among the 46 (16.1\%) patients found to have a solid cyst component on EUS, only 26 were confirmed to have IPMNs with adenocarcinoma, and 1 had IPMN with HGD. The remainder included 12 cases of IPMNs without HGD or adenocarcinoma, 4 cases of MCNs without adenocarcinoma, and 3 cases of serous cystadenomas.

\section{Diagnostic performance of AGA guidelines}

Overall, the AGA guidelines would have recommended surgery in $121(40.3 \%)$ patients and surveillance in 179 (59.7\%) patients. Among patients who would have been recommended for surgery, 45 (37.2\%) had cancer/HGD and would have been appropriately referred, whereas 76 (62.8\%) had no evidence of cancer/HGD. Among patients who would have been recommended for surveillance, 170 (95.0\%) had no evidence of cancer/HGD and would have been appropriately referred; however, $9(5.0 \%)$ patients had cancer that would have been "missed."

Overall, for the finding of cancer/HGD on surgical pathology, the AGA guidelines had a sensitivity of $83.3 \%$ (95\% confidence interval $[\mathrm{Cl}] 70.7-92.1)$, specificity of $69.1 \%$ (95\% Cl $62.9-$ 74.8), positive predictive value (PPV) of $37.2 \%$ (95\% Cl $28.6-$ 46.4), negative predictive value (NPV) of $95.0 \%$ (95\% Cl 90.7 97.7), positive likelihood ratio of 2.70 (95\% Cl 2.16-3.37), negative likelihood ratio of 0.24 (95\% Cl $0.13-0.44)$, and diagnostic accuracy of $71.7 \%$ (95\% Cl 67.4-74.6). - Table 5 shows the performance characteristics of the AGA guidelines.

\section{Missed' cancers}

There were no obvious similarities between the 9 "missed" cancers except that all had a cyst size $\geq 1 \mathrm{~cm}$. None had a solid component or dilated main pancreatic duct $\geq 5 \mathrm{~mm}$ on EUS, and only 5 had a cyst size $\geq 3 \mathrm{~cm}$. Cytology was not performed on 2 
- Table 3 Outcomes in patients undergoing surgical resection for pancreatic cystic neoplasms.

\begin{tabular}{|c|c|c|}
\hline Total $\mathbf{n}=\mathbf{3 0 0}$ & & Incidence (n, \%) \\
\hline \multirow[t]{4}{*}{ AGA high-risk features } & Cyst size $\geq 30 \mathrm{~mm}$ & $130(43.3)$ \\
\hline & Main pancreatic duct size $\geq 5 \mathrm{~mm}$ & $54(30.2)$ \\
\hline & Solid mass & $46(16.1)$ \\
\hline & Concerning cytology (cellular atypia, HGD, or cancer) & $98(34.7)$ \\
\hline \multirow[t]{4}{*}{ Number of AGA high-risk features } & 0 features & $134(44.6)$ \\
\hline & 1 feature & $113(37.7)$ \\
\hline & 2 features & $42(14.0)$ \\
\hline & 3 features & $11(3.7)$ \\
\hline \multirow[t]{3}{*}{ AGA guidelines recommend surveillance } & 0 features + negative cytology & $102(34.0)$ \\
\hline & 1 feature + negative cytology & $77(25.7)$ \\
\hline & Total surveillance & $179(59.7)$ \\
\hline \multirow[t]{7}{*}{ AGA guidelines recommend surgery } & 0 features + concerning cytology & $32(10.7)$ \\
\hline & 1 feature + concerning cytology & $36(12.0)$ \\
\hline & 2 features + negative cytology & $21(7.0)$ \\
\hline & 2 features + concerning cytology & $21(7.0)$ \\
\hline & 3 features + negative cytology & $2(0.7)$ \\
\hline & 3 features + concerning cytology & $9(3.0)$ \\
\hline & Total surgery & $121(40.3)$ \\
\hline
\end{tabular}

- Table4 Patients with malignant pancreatic cystic neoplasms.

\begin{tabular}{|l|l|l|}
\hline Total $\mathbf{n = 5 4}$ & & Incidence (n, \%) \\
\hline AGA guidelines recommend surveillance & 0 features + negative cytology & $4(7.4)$ \\
\hline & 1 feature + negative cytology & $5(9.3)$ \\
\hline & Total surveillance & $9(16.7)$ \\
\hline AGA guidelines recommend surgery & 0 features + concerning cytology & $9(16.7)$ \\
\hline & 1 feature + concerning cytology & $15(27.8)$ \\
\hline & 2 features + negative cytology & $4(7.4)$ \\
\hline & 2 features + concerning cytology & $12(22.2)$ \\
\hline 3 features + negative cytology & $2(3.7)$ \\
\hline
\end{tabular}

patients, was unsatisfactory on 1 patient, and was benign on 6 patients. On surgical pathology, there were $2 \mathrm{MCN}$ s with carcinoma and 7 IPMNs with carcinoma. The 9 "missed" cancers were all resected between 2005-2011 (2 in 2006, 3 in 2007, and 1 each in 2005, 2008, 2009, and 2011). These patients were referred for surgery based on either change in cyst size on subsequent cross-sectional imaging at 6 months to 12 months, or based on other factors not included in the AGA guidelines, such as markedly elevated cyst fluid CEA levels, being "young and fit" per Fukuoka guidelines, and patient preferences.

If our definition of "concerning" cytology had been changed to a strict definition, which only included unequivocal findings of high-grade dysplasia or adenocarcinoma, the performance of the AGA guidelines would have been less robust. There would have been 21 "missed" cancers, representing $11.7 \%$ of patients who would have been referred for surveillance. The specificity would have been $85.4 \%$ (95\% Cl 80.3-89.5), sensi- 
- Table 5 Performance characteristics of 2015 AGA guidelines.

\begin{tabular}{|c|c|c|c|}
\hline \multicolumn{4}{|l|}{ Results using 2015 AGA guidelines } \\
\hline & $\mathrm{n}=300$ patients & Cancer found $(n=54, \%)$ & No Cancer $(n=246, \%)$ \\
\hline & $\begin{array}{l}\text { Recommend surgery } \\
(n=121)\end{array}$ & $45(37.2)$ & $76(62.8)$ \\
\hline & $\begin{array}{l}\text { Recommend surveillance } \\
(n=179)\end{array}$ & $9(5.0)$ & $170(95.0)$ \\
\hline \multirow[t]{8}{*}{$\begin{array}{l}\text { Performance characteristics for finding of } \\
\text { cancer/HGD on surgical pathology }\end{array}$} & & & $\begin{array}{l}\text { Performance (\%, } 95 \% \text { confidence } \\
\text { interval) }\end{array}$ \\
\hline & & Specificity & $69.1(62.9-74.8)$ \\
\hline & & Sensitivity & $83.3(70.7-92.1)$ \\
\hline & & Positive predictive value & $37.2(28.6-46.4)$ \\
\hline & & Negative predictive value & $95.0(90.7-97.7)$ \\
\hline & & Positive likelihood ratio & $2.70(2.16-3.37)$ \\
\hline & & Negative likelihood ratio & $0.24(0.13-0.44)$ \\
\hline & & Accuracy & $71.7(67.4-74.6)$ \\
\hline
\end{tabular}

tivity $61.1 \%(95 \% \mathrm{Cl} 46.9-74.1)$, PPV $47.8 \%$ (95\% Cl 35.760.2), and NPV $90.9 \%(95 \% \mathrm{Cl} 86.4-94.3)$.

\section{Discussion}

PCNs present a significant dilemma for both patients and providers due to the risk of malignancy at time of diagnosis, variable risk of malignancy over time, and significant morbidity and potential mortality associated with surgical resection [7,9]. Despite the role of cross-sectional imaging and EUS-FNA, it continues to be challenging to accurately differentiate between PCNs and apply surveillance protocols $[10,11]$. Prior consensus guidelines, including the 2006 Sendai guidelines and the 2012 Fukuoka guidelines, have remained imperfect owing to the low quality of evidence used to develop them [15-17]. After performing a technical review of the literature [25], the AGA published guidelines for management of asymptomatic PCNs [26] call for a much less aggressive surveillance regimen than the Fukuoka guidelines. The 2015 AGA guidelines have been regarded as controversial due to their mostly conditional recommendations and overall low quality of evidence despite an extensive technical review [27-30].

In this multicenter retrospective study, we evaluated performance of the AGA guidelines in a large cohort of patients with surgically confirmed pathology. Cystic degeneration of pancreatic ductal adenocarcinomas, cystic neuroendocrine tumors, solid pseudopapillary neoplasms, and main duct IPMNs were not included in our study, because these lesions are typically recommended to undergo surgery and are not addressed by the AGA guidelines. In our evaluation of 300 patients who underwent surgical resection for PCNs relevant to the AGA guidelines, adherence to these guidelines reduced the number of patients who would have undergone surgical resection by approximately $60 \%$.

This reduction in patients referred for surgery is due to the fact that only $53 / 300(17.7 \%)$ patients met 2 or more high-risk features as classified by the AGA guidelines, and only 98/300 (34.6\%) patients had concerning cytology findings. The AGA guidelines would have recommended surgery in only $121 / 300$ $(40.3 \%)$ patients, in whom only 45 cysts harbored adenocarcinoma or HGD on surgical pathology. Notably, 21/45 (46.7\%) cysts demonstrated 2 or more high-risk features with or without concerning cytology findings, whereas the remaining 24 (53.3\%) cysts had fewer than 2 high-risk features but had concerning cytology findings. This suggests that the use of 2 or more high-risk features alone per the AGA guidelines is insufficient, and that there remains a role for EUS and cytology, despite its reportedly poor sensitivity [11]. In addition, in clinical practice these patients also often had other EUS findings or patient-specific factors that led to surgical referral. Given that the definitions for "concerning" cytology are highly variable, this finding demonstrates that cytology results can dramatically influence the clinical decision for surgery versus surveillance. To highlight this issue, "atypical" cytology was used in our study given that in our clinical experience, "atypical” cytology results are often accompanied by additional commentary from the pathologist such as "cannot rule out" advanced dysplasia or welldifferentiated adenocarcinoma.

Our study validates the major strengths of the AGA guidelines in that approximately $60 \%$ of patients would have been spared from undergoing surgical resection, with a high sensitivity (83.3\%) and NPV (95.0\%). However, despite the apparent advantage in the accurate triage of low-risk PCNs, the AGA guidelines remain imperfect. Of the cysts with fewer than 2 high-risk features and no evidence of concerning cytology, 
which would have been triaged to surveillance per the AGA guidelines, 9 cancers would have been "missed." Interestingly, all 9 patients had their tumors resected prior to 2011, although 4 would have been missed under the Sendai guidelines. Our results may actually underestimate the true performance characteristics of the AGA guidelines, specifically with regards to sensitivity and NPV given advances in imaging technology over our 10 -year study period.

A recent retrospective study suggested that both the original Sendai guidelines and the Fukuoka guidelines are able to accurately determine which patients with pancreatic cysts have advanced neoplasia, with the Sendai guidelines demonstrating 91.7\% sensitivity, $21.5 \%$ specificity, $21 \%$ PPV, and $91.9 \%$ NPV for identification of advanced neoplasia [24]. However, as demonstrated in prior studies [19], several patients with high-grade dysplasia were missed. While we did not directly compare the AGA, Sendai, and Fukuoka guidelines, the observation that the 9 cancers in our surgical cohort were triaged to surveillance by the AGA guidelines but resected per the Sendai guidelines attests to the higher sensitivity but lower specificity of the Sendai guidelines.

Our study follows several others that have been published evaluating the AGA guidelines. Singhi et al reported a retrospective study of the AGA guidelines applied to 225 patients with pancreatic cystic lesions, of whom only 41 patients had surgical pathology [31]. Notably, $45 \%$ of IPMNs with HGD or adenocarcinoma were triaged to surveillance. Among all pancreatic cysts, the AGA guidelines had $62 \%$ sensitivity, $79 \%$ specificity, 57\% PPV, and 87\% NPV for identification of advanced neoplasia. Similarly, Ma et al reported in a retrospective study that the AGA guidelines did not appear to be superior to Fukuoka in identifying advanced neoplasia in suspected PCNs [32]. In their study, both guidelines had PPV of $71 \%$ to $74 \%$ and would lead to unnecessary resections in patients without advanced neoplasia, while missing additional cases with advanced neoplasia. The precise reasons for discordance among studies are unclear and may be due to differences in study populations and data analysis.

Prior studies evaluating the Sendai and Fukuoka guidelines have suggested that although cyst surveillance is cost-effective when compared to no surveillance or immediate surgery [33], ongoing cyst surveillance still confers significant additional costs to society when applied to large populations of patients who have asymptomatic PCNs [28]. While a formal cost analysis was not performed as part of this study, adherence to the AGA guidelines would have likely resulted in substantial cost savings given that almost $60 \%$ of patients would have been triaged to surveillance rather than surgical resection.

There are several limitations of this study that merit discussion. The majority of our patients were referred from outside institutions and therefore follow-up was limited. The retrospective nature of our study also made it impossible to determine how many of our patients were truly asymptomatic. Furthermore, the natural history of those patients recommended to undergo surveillance by the AGA guidelines is not known, as all patients underwent surgical resection.
Importantly, our study population was highly selected to include only those patients who underwent EUS and subsequently had surgical resection for non-main duct IPMNs, MCNs, and SCAs. While these are the lesions that were specifically addressed by the AGA guidelines, we acknowledge that often the pathology is not known until surgical resection, and the AGA guidelines also do not recommend EUS for every patient. Nevertheless, by biasing our study population away from the lowest- and highest-risk lesions, both of which would have never undergone EUS, we are still able to demonstrate that only $5 \%$ of patients recommended to undergo surveillance were found to have cancer on pathology. This finding suggests that when the AGA guidelines are applied to a broader population of predominantly low-risk asymptomatic PCNs, the actual rate of missed cancer is likely much lower. Furthermore, although $5 \%$ of patients recommended to undergo serial surveillance were ultimately found on pathology to have cancer, this does not necessarily imply outright missing a diagnosis of cancer, as these PCNs would have potentially developed changes or concerning features on surveillance that would prompt surgical referral.

\section{Conclusion}

In conclusion, in our large multicenter surgical cohort, adherence to the 2015 AGA guidelines would have resulted in nearly $60 \%$ fewer patients being referred for surgical resection and accurately recommended surveillance for most patients with asymptomatic PCNs. However, a malignancy was identified in $5 \%$ of patients for whom surveillance was recommended, although these cases may have been subsequently identified during surveillance. Our study was specifically performed as an audit of real world clinical practice at multiple major academic institutions, and highlights a continued role for EUS-FNA in the management of a select population of higher-risk PCNs. While current studies are exploring molecular markers [31,34], as well as developing nomograms for predicting the probability of high-risk PCNs [35], our data emphasize the continued need to individualize treatment of PCNs until additional higher-quality prospective multicenter studies are performed.

\section{Competing interests}

None

\section{References}

[1] Kimura W, Nagai H, Kuroda A et al. Analysis of small cystic lesions of the pancreas. Int J Pancreatol 1995; 18: 197-206

[2] Spinelli KS, Fromwiller TE, Daniel RA et al. Cystic pancreatic neoplasms: observe or operate. Ann Surg 2004; 239: 651 - 657

[3] Al-Haddad M, Schmidt MC, Sandrasegaran K et al. Diagnosis and treatment of cystic pancreatic tumors. Clin Gastroenterol Hepatol 2011; 9: 635-648

[4] Sarr MG, Murr M, Smyrk TC et al. Primary cystic neoplasms of the pancreas. Neoplastic disorders of emerging importance-current 
state-of-the-art and unanswered questions. J Gastrointest Surg 2003; 7: $417-428$

[5] Zamboni G, Scarpa A, Bogina G et al. Mucinous cystic tumors of the pancreas: clinicopathological features, prognosis, and relationship to other mucinous cystic tumors. Am J Surg Pathol 1999; 23: 410-422

[6] Balcom JHt, Rattner DW, Warshaw AL et al. Ten-year experience with 733 pancreatic resections: changing indications, older patients, and decreasing length of hospitalization. Arch Surg 2001; 136: 391 - 398

[7] Kamata K, Kitano M, Kudo M et al. Value of EUS in early detection of pancreatic ductal adenocarcinomas in patients with intraductal papillary mucinous neoplasms. Endoscopy 2014; 46: 22 - 29

[8] Sedlack R, Affi A, Vazquez-Sequeiros E et al. Utility of EUS in the evaluation of cystic pancreatic lesions. Gastrointest Endosc 2002; 56: $543-547$

[9] Brugge WR, Lewandrowski K, Lee-Lewandrowski E et al. Diagnosis of pancreatic cystic neoplasms: a report of the cooperative pancreatic cyst study. Gastroenterology 2004; 126: 1330 - 1336

[10] Ahmad NA, Kochman ML, Lewis JD et al. Can EUS alone differentiate between malignant and benign cystic lesions of the pancreas? Am J Gastroenterol 2001; 96: 3295-3300

[11] Centeno BA, Warshaw AL, Mayo-Smith W et al. Cytologic diagnosis of pancreatic cystic lesions. A prospective study of 28 percutaneous aspirates. Acta Cytol 1997; 41: 972 - 980

[12] Gaddam S, Ge PS, Keach JW et al. Suboptimal accuracy of carcinoembryonic antigen in differentiation of mucinous and nonmucinous pancreatic cysts: results of a large multicenter study. Gastrointest Endosc 2015; 82: 1060 - 1069

[13] Singhi AD, Nikiforova MN, Fasanella KE et al. Preoperative GNAS and KRAS testing in the diagnosis of pancreatic mucinous cysts. Clin Cancer Res 2014; 20: $4381-4389$

[14] Lubezky N, Loewenstein S, Ben-Haim M et al. MicroRNA expression signatures in intraductal papillary mucinous neoplasm of the pancreas. Surgery 2013; 153: 663-672

[15] Tanaka M, Chari S, Adsay V et al. International consensus guidelines for management of intraductal papillary mucinous neoplasms and mucinous cystic neoplasms of the pancreas. Pancreatology 2006; 6: $17-32$

[16] Tanaka M, Fernandez-del Castillo C, Adsay V et al. International consensus guidelines 2012 for the management of IPMN and MCN of the pancreas. Pancreatology 2012; 12: $183-197$

[17] Del Chiaro M, Verbeke C, Salvia R et al. European experts consensus statement on cystic tumours of the pancreas. Dig Liver Dis 2013; 45 : $703-711$

[18] Anand N, Sampath K, Wu BU. Cyst features and risk of malignancy in intraductal papillary mucinous neoplasms of the pancreas: a meta-analysis. Clin Gastroenterol Hepatol 2013; 11: 913-921

[19] Nguyen AH, Toste PA, Farrell JJ et al. Current recommendations for surveillance and surgery of intraductal papillary mucinous neoplasms may overlook some patients with cancer. J Gastrointest Surg 2015; 19: $258-265$

[20] Goh BK, Tan DM, Thng CH et al. Are the Sendai and Fukuoka Consensus Guidelines for Cystic Mucinous Neoplasms of the pancreas useful in the initial triage of all suspected pancreatic cystic neoplasms? A single-institution experience with 317 surgically-treated patients Ann Surg Oncol 2014; 21: 1919-1926

[21] Pelaez-Luna M, Chari ST, Smyrk TC et al. Do consensus indications for resection in branch duct intraductal papillary mucinous neoplasm predict malignancy? A study of 147 patients Am J Gastroenterol 2007; 102: $1759-1764$

[22] Fritz S, Klauss M, Bergmann F et al. Small (Sendai negative) branchduct IPMNs: not harmless. Ann Surg 2012; 256: 313-320

[23] Sawhney MS, Al-Bashir S, Cury MS et al. International consensus guidelines for surgical resection of mucinous neoplasms cannot be applied to all cystic lesions of the pancreas. Clin Gastroenterol Hepatol 2009; 7: $1373-1376$

[24] Kaimakliotis P, Riff B, Pourmand K et al. Sendai and Fukuoka Consensus Guidelines identify advanced neoplasia in patients with suspected mucinous cystic neoplasms of the pancreas. Clin Gastroenterol Hepatol 2015; 13: $1808-1815$

[25] Scheiman JM, Hwang JH, Moayyedi P. American gastroenterological association technical review on the diagnosis and management of asymptomatic neoplastic pancreatic cysts. Gastroenterology 2015; 148: $824-848$

[26] Vege SS, Ziring B, Jain R et al. American gastroenterological association institute guideline on the diagnosis and management of asymptomatic neoplastic pancreatic cysts. Gastroenterology 2015; 148: $819-822$

[27] Fernandez-Del Castillo C, Tanaka M. Management of pancreatic cysts: the evidence is not here yet. Gastroenterology 2015; 148: 685-687

[28] Canto MI, Hruban RH. Managing pancreatic cysts: less is more? Gastroenterology 2015; 148: 688-691

[29] Moayyedi P, Weinberg DS, Schunemann $\mathrm{H}$ et al. Management of pancreatic cysts in an evidence-based world. Gastroenterology 2015; 148: $692-695$

[30] Harris RP. Incidental findings in the pancreas (and elsewhere): putting our patients (and ourselves) in a difficult situation. Ann Intern Med 2015; $162: 787-789$

[31] Singhi AD, Zeh H], Brand RE et al. American Gastroenterological Association guidelines are inaccurate in detecting pancreatic cysts with advanced neoplasia: a clinicopathologic study of 225 patients with supporting molecular data. Gastrointest Endosc 2016; 83: 1107 1117

[32] Ma GK, Goldberg DS, Thiruvengadam N et al. Comparing American Gastroenterological Association Pancreatic Cyst Management Guidelines with Fukuoka Consensus Guidelines as predictors of advanced neoplasia in patients with suspected pancreatic cystic neoplasms. J Am Coll Surg 2016; 223: 729-737

[33] Huang ES, Gazelle GS, Hur C. Consensus guidelines in the management of branch duct intraductal papillary mucinous neoplasm: a costeffectiveness analysis. Dig Dis Sci 2010; 55: 852-860

[34] Springer S, Wang Y, Dal Molin M et al. A Combination of Molecular Markers and Clinical Features Improve the Classification of Pancreatic Cysts. Gastroenterology 2015; 149: 1501 - 1510

[35] Shimizu Y, Yamaue H, Maguchi H et al. Validation of a nomogram for predicting the probability of carcinoma in patients with intraductal papillary mucinous neoplasm in 180 pancreatic resection patients at 3 high-volume centers. Pancreas 2015; 44: 459-464 